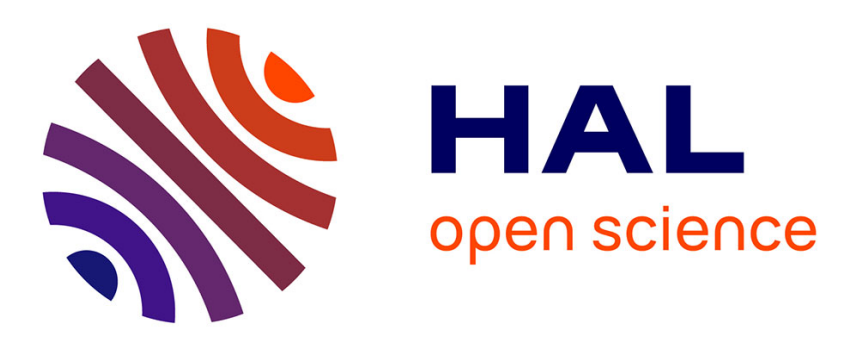

\title{
Critical study of the Josephson oscillator in frequency conversion and detection
}

\author{
G. Vernet, R. Adde
}

\section{To cite this version:}

G. Vernet, R. Adde. Critical study of the Josephson oscillator in frequency conversion and detection. Revue de Physique Appliquée, 1975, 10 (3), pp.165-168. 10.1051/rphysap:01975001003016500 . jpa00243896

\section{HAL Id: jpa-00243896 https://hal.science/jpa-00243896}

Submitted on 1 Jan 1975

HAL is a multi-disciplinary open access archive for the deposit and dissemination of scientific research documents, whether they are published or not. The documents may come from teaching and research institutions in France or abroad, or from public or private research centers.
L'archive ouverte pluridisciplinaire HAL, est destinée au dépôt et à la diffusion de documents scientifiques de niveau recherche, publiés ou non, émanant des établissements d'enseignement et de recherche français ou étrangers, des laboratoires publics ou privés. 


\title{
Classification \\ Physics Abstracts 8.440 \\ CRITICAL STUDY OF THE JOSEPHSON OSCILLATOR IN FREQUENCY CONVERSION AND DETECTION (*)
}

\author{
G. VERNET and R. ADDE \\ Institut d'Electronique Fondamentale $(* *)$ \\ Bâtiment 220, Université Paris XI, 91405 Orsay, France
}

(Reçu le 6 janvier 1975, accepté le 22 janvier 1975)

\begin{abstract}
Résumé. - On présente des observations relatives à l'utilisation de l'oscillateur-mélangeur Josephson comme détecteur hétérodyne. La perturbation de l'oscillateur Josephson pảr un signal extérieur et les effets de synchronisation sont étudiés. Les conditions dans lesquelles l'oscillateurmélangeur Josephson est susceptible de devenir un détecteur d'ondes submillimétriques compétitif sont également analysées.
\end{abstract}

Abstract. - We discuss topics related to the Josephson oscillator mixer as an heterodyne detector. Perturbation of the Josephson oscillator by an external signal and phase locking effects are studied. The conditions where the Josephson oscillator-mixer may be a competitive submillimetric detector are also considered.

1. Introduction. - Josephson devices may be operated as oscillator-mixer to realize heterodyne detection. In this mode of operation, mixing of an external radiation with the Josephson self-oscillation is performed in the same device and it has been shown that the observation of frequency conversion signals is not straightforward $[1,2]$. In mixing and detection applications the characteristics of the Josephson selfoscillation [3, 4] and its perturbation by the external radiation $[5,6]$ to be detected are important factors which will be discussed here. The conditions where the Josephson oscillator-mixer may be a competitive detector are also considered.

2. The Josephson oscillator. - For very high frequency applications, the best Josephson device is actually the superconducting point contact due to its low capacitance and relatively better coupling in the infrared region.

A point contact Josephson oscillator not coupled to an external cavity has a rather broad linewidth $\Delta f_{0}[4,7]$ typically between $10 \mathrm{MHz}$ and $1 \mathrm{GHz}$ depending of the dynamic resistance $R_{\mathrm{D}}$ and the total direct current $I_{\mathrm{dc}}$. Consequently the available power $P$ in a narrow bandwidth $B \ll \Delta f_{0}$ is strongly reduced since $P=P_{0}\left(B / \Delta f_{0}\right)$ where $P_{0}$ is the total power radiated by the contact.

When such a device is used as an oscillator-mixer to detect monochromatic radiation, a narrow band detec-

(*) Ce travail se rapporte à la Thèse de Doctorat d'Etat de G. Vernet (ref. C. N. R. S. A. O. 11 233).

$(* *)$ Laboratoire associé au C. N. R. S. tion cannot be performed with good sensitivity performance. This situation is represented figure $1 a-b$. The monochromatic external radiation at $\left(f_{0}+f_{\mathrm{i}}\right)$ is mixed with the Josephson oscillation of linewidth $\Delta f_{0}$. The detected power in the bandwidth $B$ of the intermediate frequency $f_{\mathrm{i}}$ can be written [2]

$$
P_{f \mathrm{i}}(B)=S \frac{P_{0}}{\Delta f_{0}} B P_{\mathrm{e}}
$$

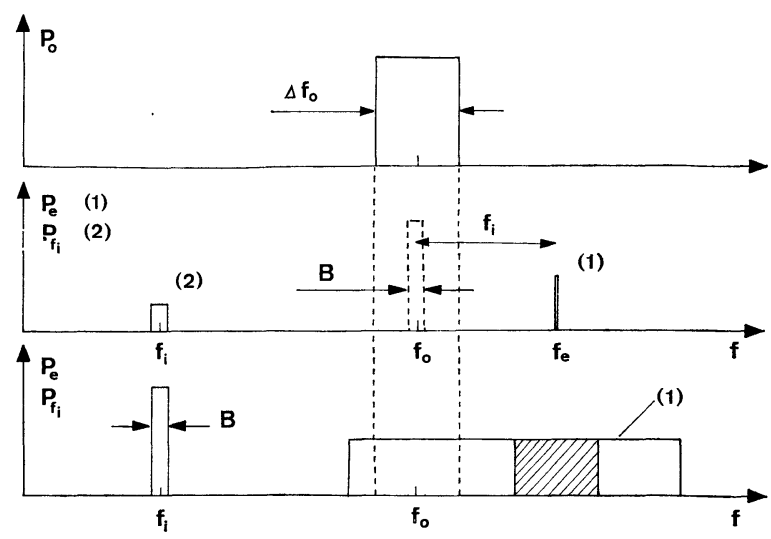

FIG. 1. - a) Schematized power spectral density of the Josephson oscillation at frequency $f_{0}$. The linewidth $\Delta f_{0}$ is strongly dependent of the d. c. parameters of the junction. A typical value is $\Delta f_{0} \sim 1 \mathrm{GHz}$ for a normal resistance junction of $10 \Omega$. b) Mixing of the Josephson oscillation with a monochromatic external radiation of frequency $f_{\mathrm{e}}$ in a bandwidth $\mathrm{B}$. Only a small fraction of the total Josephson power is used when $B \ll \Delta f_{0}$ as in experiments with small intermediate frequency $f_{\mathbf{i}}\left(f_{\mathrm{i}} \lesssim\right.$ $100 \mathrm{MHz}$ ) c) Mixing of the Josephson oscillation with a relatively wide band external signal $\left(\Delta f_{\mathrm{e}} \gtrsim \Delta f_{0}\right)$. In this case all the Josephson power is used and the detected power at the i. f. increases considerably. 
where $P_{\mathrm{e}}$ is the low level external power and $S$ a factor including the conversion losses at $f_{\mathrm{i}}$ and $f_{\mathrm{e}}$. For example a $10 \Omega$ Josephson junction has a natural linewidth $\Delta f_{0} \gtrsim 1 \mathrm{GHz}$ at $1 \mathrm{mV}\left(f_{0} \simeq 500 \mathrm{GHz}\right)$ which means that a selective detection with an oscillator-mixer of intermediate frequency $100 \mathrm{MHz}$ and a $1 \mathrm{MHz}$ bandwidth will use only $10^{-3}$ of the available Josephson power. Therefore a much larger bandwidth $B$ should be used to optimize sensitivity performance and this implies a large intermediate frequency $f_{\mathbf{i}}$.

3. Perturbation of the Josephson oscillator. - An important factor to be taken into account for the operation of the Josephson oscillator-mixer is the possible phase synchronization of the Josephson oscillation with the external radiation. Several parameters need to be considered : the external signal power $P_{\mathrm{e}}$, the frequency difference $\left|f_{0}-f_{1}\right|=\delta f=f_{\mathrm{i}}$ which is the intermediate frequency, and external stabilization of the Josephson oscillation.

3.1 INFLUENCE of $\boldsymbol{P}_{\mathrm{e}}$ AND $\delta f$. - Figure 2 shows the influence of external radiation of frequency $f_{1}$ on a $\mathrm{Nb}-\mathrm{Nb}$ point contact Josephson oscillator at $\mathrm{X}$ band [6]. As the unperturbed Josephson linewidth figure $2 a$ is relatively broad ( $\simeq 200 \mathrm{MHz}$ ) the emission level cannot be measured by sweeping the receiver frequency. It is detected with a constant receiver frequency $f_{\text {det }}$ while sweeping the $V I$ characteristic. Two cases must be considered which correspond respectively to situations where the operating point on the VIC such that $f_{0}=f_{\text {det }}$ is far from the step induced by the external signal at $f_{1}$ (Fig. $2 b$ ) or is in the curvature of this step (Fig. 2c), that is $\delta f=\left|f_{\text {det }}-f_{1}\right|$ is relatively large or small.

We have shown [6] when $\delta f$ is large that the perturbation of the Josephson oscillation manifests itself in a progressive decrease of the emission level and an increase in linewidth which are well related to modifications of the VIC parameters $R_{\mathrm{D}}$ and $I$.

On the contrary for small $\delta f$, the emission level at $f_{\text {det }}$ drops sharply when the step appears in the $V I C$ and there is no more correlation with the VIC parameters. In this latter case it is seen that some energy associated to the Josephson oscillation at $f_{0}=f_{\text {det }}$ has vanished due to a progressive phase locking of the Josephson radiation on the external signal. Figure 3 indicates clearly that this effect increases when $\delta f$ is decreased. No visible effect on the Josephson oscillation was observed before a tiny step becomes visible in the VIC.

This partial phase locking which makes the Josephson emission level drop explains why frequency conversion signals were not observed in our previous experiments [2] with a small i. f. of $100 \mathrm{MHz}$ and moderate external monochromatic radiation power.

The experimental results represented in figures 4 and 5 give a good visualization of the progressive phase locking which occurs in point contacts irradiated with an external signal. When such a device is irradiated at a

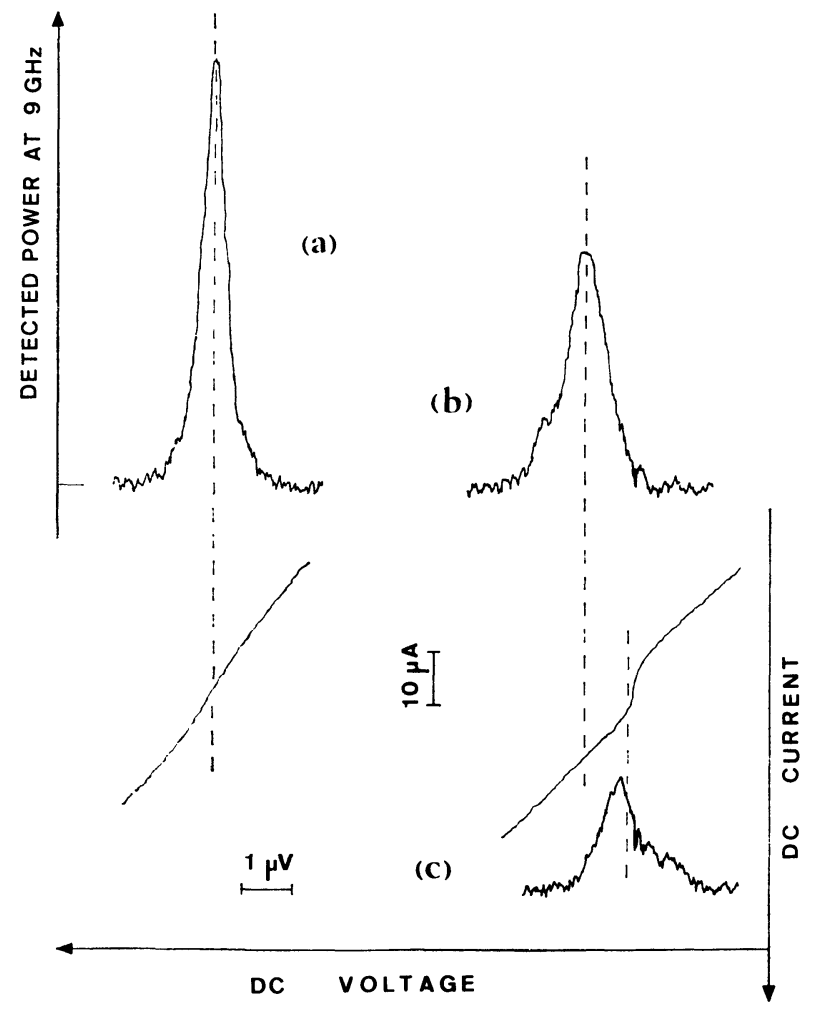

FIG. 2. - Perturbation of the Josephson emission line by an external radiation $\left(f_{1}\right)$. The emitted radiation is detected with a receiver tuned at $f_{\text {det }}$ while sweeping the $V-I$ characteristic. The vertical dotted lines indicate $f_{\mathrm{det}}$. If $P_{1}=0$, the natural Josephson linewidth is measured $a$ ). For the same contact conditions and $P_{1}=-30 \mathrm{dBs}, b$ ) corresponds to $\left.\delta f=\left|f_{1}-f_{\text {det }}\right|=500 \mathrm{MHz}, c\right)$ corresponds to

$$
\delta f=\left|f_{1}-f_{\text {det }}\right|=20 \mathrm{MHz} .
$$

The $V I$ characteristics corresponding respectively to $a$ ) and $b$ ), $c$ ) are also represented. Comparison of $b$ ) and $c$ ) clearly shows the strong difference in detected power for large and small $\delta f$.

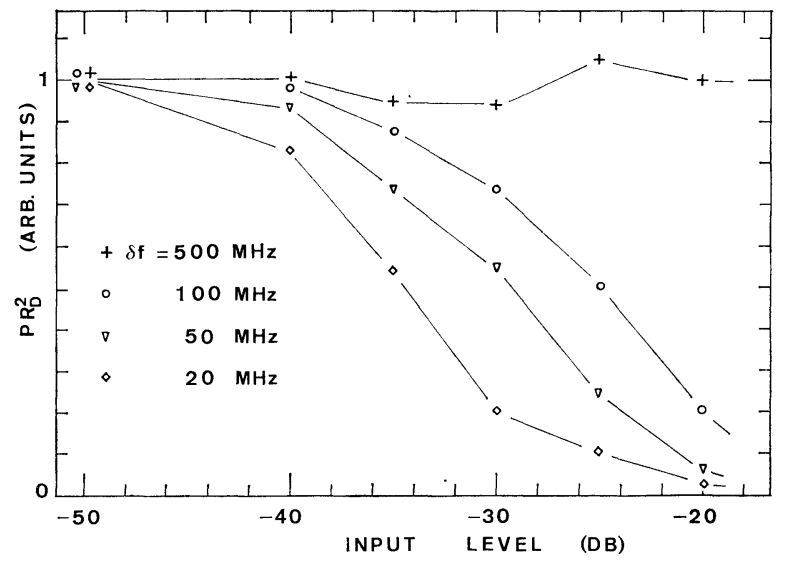

FIg. 3. - Evolution as a function of the incident power $\boldsymbol{P}_{1}$ of the quantity $P R_{\mathrm{D}}^{2}$ where $P$ is the maximum radiated power at $f_{0}=f_{\text {det }}$ and $R_{\mathrm{D}}$ the corresponding dynamic resistance. The drop of $P R_{\mathrm{D}}^{2}$ with increasing $P_{1}$ becomes more important as the parameter $\delta f=\left|f_{1}-f_{\text {det }}\right|$ is reduced. The calibration is the same as in figure 2.

frequency $f_{1}$, a substep often appears in the $V I C$ at $f_{1} / 2$. We have studied the influence of the substep on the Josephson oscillation for different $\delta f=\left|f_{1} / 2-f_{0}\right|$, i. e. 


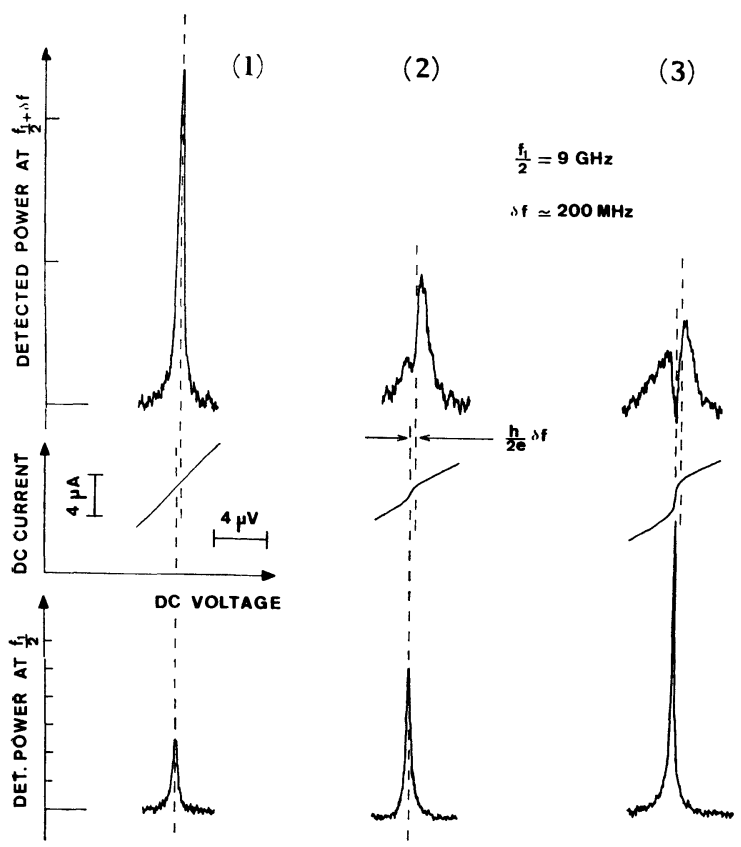

(b)

FIG. 4. - Phase locking of the Josephson oscillator with an external radiation at $f_{1}$ through the second harmonic of the fundamental oscillation $\left(f_{1} \simeq 2 f_{0}\right)$. a) VIC around the operating point at $f_{1} / 2$ for zero external power $P_{\mathrm{e}}(1)$ and increasing power $P_{\mathrm{e}}(2$ and 3$)$ showing small step appearing at $f_{1} / 2$. b) From (1) to (3) the steep decrease due to phase-locking of the Josephson radiated power when the contact is biased at $f_{1} / 2+\delta f$ in the rounding of the step. c) Corresponding growth of the statistical weight of the Josephson current components at $f_{1} / 2$ with increasing phase-locking $(\delta f=0)$.

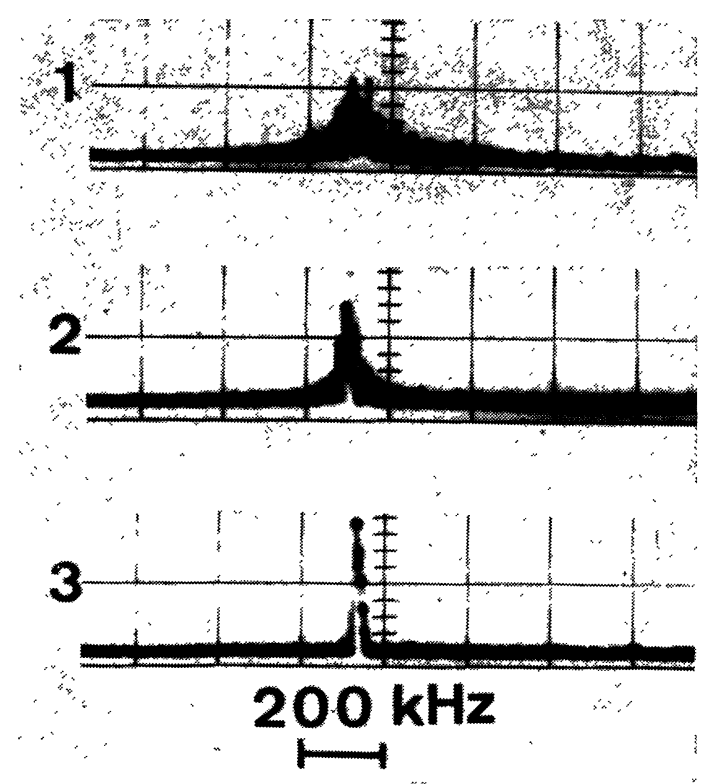

FIG. 5. - Phase locking of a point contact Josephson oscillator at $f_{0}=9 \mathrm{GHz}$ on an external radiation at $f_{1}=2 f_{0}$ of increasing power (from 1 to 3). In case (3) phase locking is total and the Josephson linewidth is smaller than the $20 \mathrm{kHz}$ bandwith of the receiver.

the phase locking on the external radiation via the second harmonic component in the Josephson current. Figure $4 b$ corresponds to a situation where a point contact is biased in the rounding of the step $\frac{1}{2}$ such that $\delta f=200 \mathrm{MHz}$. As the step appears, the steep decrease of the Josephson detected level at $f_{\text {det }}$ shows again that when the Josephson oscillator is tuned in the rounding of the step $\frac{1}{2}$, it becomes progressively locked on the external signal with increasing power $P_{\mathrm{e}}$. In other words the statistical weight of the Josephson oscillation components around $f_{0}=f_{\text {det }}$ drops and these components are displaced by phase-locking at the frequency $f_{1} / 2$. This latter effect is represented in figure $4 c$ with the receiver tuned at $f_{1} / 2$. For $P_{\mathrm{e}}=0$, the unperturbed Josephson line is detected (case 1), while for increasing $\boldsymbol{P}_{\mathrm{e}}$, the amplitude of the detected level increases and its linewidth decreases (cases 2 and 3). Figure 5 shows that for a large substep amplitude, a strong phase locking occurs such that the observed linewidth is smaller that the receiver bandwidth $(<20 \mathrm{kHz})$.

\subsection{Perturbation of a PHASe-LOcked Josephson} OSCILLATOR. - We consider now the situation where a Josephson oscillator which is phase locked at a frequency $f_{1} / 2$ on a first radiation $f_{1}$ as described above is subjected to a second radiation of frequency $f_{2}$ slightly different from $f_{1} / 2$. This allows us to investigate in which conditions it becomes locked on the second radiation at $f_{2}$. The Josephson linewidth at $f_{1} / 2$ is smaller than $20 \mathrm{kHz}$ and the frequency difference is $f_{2}-\left(f_{1} / 2\right)=700 \mathrm{kHz}$. Depending on the incoming power and the bias point on the $V I C$, figure 6 shows the two possible situations :

a) The Josephson oscillation remains locked at $f_{1} / 2$ without perturbation of amplitude or linewidth.

b) The signal at $f_{1} / 2$ disappears completely and a symmetrical signal appears at a frequency $f_{1} / 2+\delta f=f_{1}-f_{2}$. Now the Josephson oscillation is locked on the signal at $f_{2}$ and the signal at $f_{1}-f_{2}$ is due to mixing occuring between the Josephson radiation now locked at $f_{2}$ and the signal at $f_{1}$.

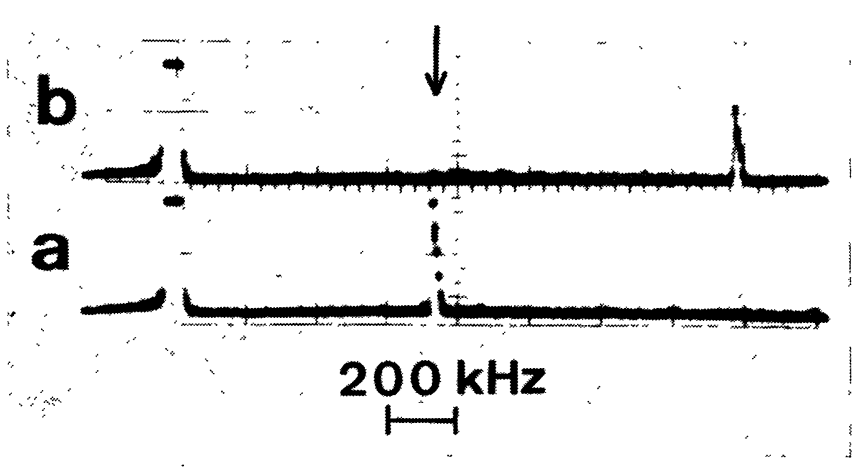

FIG. 6. - Perturbation of a phase-locked Josephson oscillator at $f_{0}=f_{1} / 2$ by an external radiation of frequency $f_{2}=f_{0}-\delta f$ (left signal). Depending of the incoming power at $f_{2}$ and of the bias point on the VIC two situations may occur : $a$ ) The Josephson oscillator remains locked at $f_{1} / 2$ (indicated by an arrow) without perturbation of level or linewidth. $b$ ) The Josephson oscillator becomes locked on the signal at $f_{2}$ and there is no more visible signal at $f_{1} / 2$. The symmetrical signal on the right is due to mixing between the Josephson oscillation at $f_{2}=f_{0}$ and the signal at $f_{1}$. 
The frequency pulling which happens between cases $a$ ) and $b$ ) must be accompanied by a variation of the voltage so that the Josephson frequency-voltage relation still holds. This is shown on figure 7 where a larger

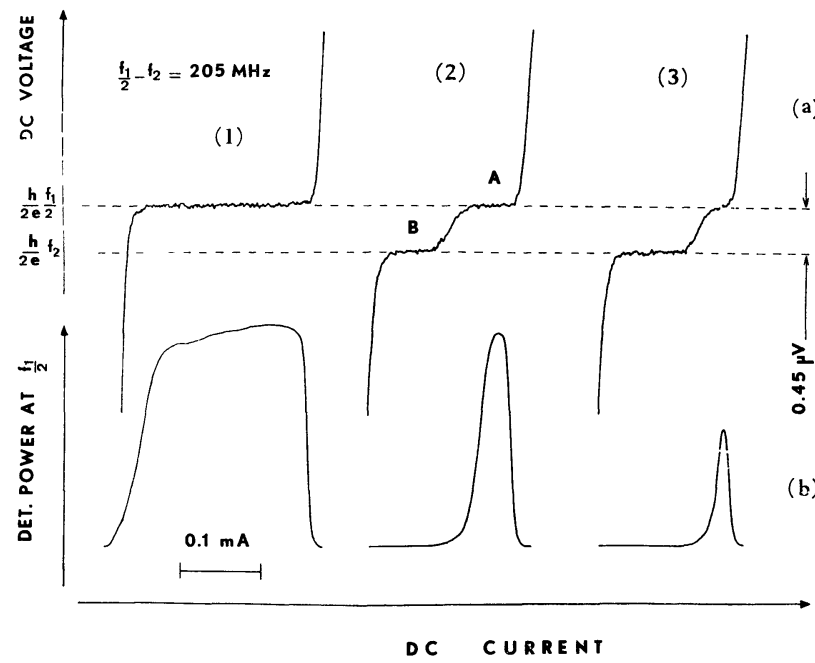

FIG. 7. - Recording on the $V I C$ and of the radiated power at $f_{1} / 2$. 1) The Josephson oscillator is locked at $f_{1} / 2$ and consequently a step appears in the $V I C$ at a voltage corresponding to this frequency. 2) The phase locked Josephson oscillator is perturbed by an external radiation at $f_{2}=f_{1} / 2+\delta f$ and a new step appears at a voltage corresponding to $f_{2}$. In (A) the detected power at $f_{1} / 2$ from the oscillator is unaffected by the signal at $f_{2}$. In (B) the oscillator is phase locked on the signal at $f_{2}$. 3) For higher power levels of the signal at $f_{2}$ the Josephson oscillator remains practically always locked at $f_{2}$.

$\delta f$ has been used for a better illustration. At the dc current bias where the Josephson detected level at $f_{1} / 2$ becomes zero if the radiation at $f_{2}$ is applied, one clearly observes an induced step at a voltage $\left(h f_{2} / 2 e\right)$ Figure $7 a$ also shows the relative degree of steepness of the transition between the two possibles situations, which is limited by the intrinsic noise which rounds the steps. When the contact is biased in this region, the system jumps back and forth between the two cases.

4. The Josephson oscillator-mixer as a detector. For very low external signal powers (before synchronization occurs), the application of the Josephson oscillator-mixer as a very high frequency heterodyne detector is in principle only limited by the large Josephson emission linewidth associated with the weak power of this local oscillator. In part 1 , we have seen that as a consequence the detection of a monochromatic radiation with a small i. f. is practically excluded.

The situation is different if the detection of non monochromatic signals is performed, that is detection of signals with a linewidth larger than the Josephson linewidth itself. This situation is represented in figure $1 c$. The detectable i. f. power in the bandwidth $B$ may be written.

$$
P_{\mathrm{i} f}(B)=\int_{\Delta f_{\mathrm{o}}} S \frac{P_{0}}{\Delta f_{0}} B \frac{\mathrm{d} P_{\mathrm{e}}}{\mathrm{d} f} \mathrm{~d} f=S P_{0} \frac{\mathrm{d} P_{\mathrm{e}}}{\mathrm{d} f} B
$$

if $\frac{\mathrm{d} P_{\mathrm{e}}}{\mathrm{d} f}$ is constant. This result depends only on the total Josephson power and it is necessary to have $B$ as large as possible since the signal to noise ratio is proportional to $\sqrt{B}$.

Recent experimental results [8] seem to confirm these predictions, with the detection of weak wideband signals $(206 \pm 10 \mathrm{GHz})$. The point contact used in this study had an emission linewidth which can be evaluated to $1 \mathrm{GHz}$, the intermediate frequency was $280 \mathrm{MHz}$ and the receiver bandwidth $400 \mathrm{MHz}$. The noise temperatures of these type of receivers should be smaller than the noise temperature of Schottky millimeter receivers if the impedance matching problem of point contact was solved. The selectivity of such detectors would also be reasonable $(\sim 100 \mathrm{MHz}$ to $1 \mathrm{GHz}$ ) which corresponds in the infrared wavelength region to a quality factor of $10^{3}-10^{4}$.

5. Conclusion. - The Josephson oscillator-mixer with its low power level in a relatively large frequency bandwidth and the phase-locking effects between the Josephson oscillation and the external signal does not compete with superconducting heterodyne receivers with an external oscillator for monochromatic radiation detection.

However it presents advantages as a relatively narrow band infrared detector if the impedance matching to the external signal can be performed. It has great simplicity since frequency tuning is performed by a proper selection of the dc bias. Its bandwidth $(\sim 1 \mathrm{GHz})$ matches the specifications in some applications particularly in infrared astronomy.

Acknowledgments. - The authors are much indebted to D. Bouchon for his technical assistance.

\section{References}

[1] LongaCre, A., Applied Superconductivity Conference, Annapolis, Maryland, IEEE, New York, 1972 p. 712.

[2] Vernet, G. and Adde, R., J. Appl. Phys. 45 (1974) 2678.

[3] Dahm, A. J., Denenstein, A., Langenberg, D. N., ParKER, W. H., Rogovin, D. and Scalapino, D. J., Phys. Rev. Lett. 22 (1969) 1416.

[4] Vernet, G. and Adde, R., Appl. Phys. Lett. 19 (1971) 195.

[5] Stancampiano, C. V. and Shapiro, S., Appl. Phys. Lett. 25 (1974) 315.

[6] Vernet, G. and Adde, R., Applied Superconductivity Conference, Chicago (Sept. 30, 1974).
[7] Note : The theoretical expression for the Josephson oscillation bandwidth is

$$
\Delta f=2 \pi\left(\frac{2 e}{h}\right)^{2} R_{\mathrm{D}}^{2}\left[I_{\mathrm{q}} \operatorname{coth}\left(\frac{e V_{0}}{2 k T}\right)+2 I_{\mathrm{p}} \operatorname{coth}\left(\frac{e V_{0}}{k T}\right)\right] .
$$

In the very high frequency limit

$$
\Delta f \rightarrow 2 \pi\left(\frac{2 e}{h}\right)^{2} R_{\mathrm{D}}^{2} I_{\mathrm{dc}} .
$$

[8] Avakjan, R. S., Vystavkin, A. N., Gubankov, V. N., Migulin, V. V. and Shtykov, V. D., Applied Superconductivity Conference, Chicago (Sept. 30, 1974). 